\title{
Avaliação radiográfica do colapso sagital na fratura toracolombar tipo B de Magerl
}

Radiographic evaluation of sagittal collapse in Magerl type B thoracolumbar fractures

\author{
Evaluación radiográfica de la cifosis en los portadores de fracturas \\ del subtipo B21 de Magerl
}

\author{
Adriano Passaglia Esperidião' \\ Ricardo Shigueaki Galhego Umeta² \\ Maria Fernanda Silber Caffaro ${ }^{3}$ \\ Robert Meves ${ }^{4}$ \\ Elcio Landim 5 \\ Osmar Avanzi ${ }^{6}$
}

\section{RESUMO}

Introdução: a cifose pós-traumática é uma complicação do tratamento conservador da fratura toracolombar tipo flexo-distrativa de Magerl (B21). A maioria das séries avalia todos os subtipos de Magerl e discute seus achados com base na perda média de cifose no acompanhamento. Objetivo: avaliar o desfecho radiográfico quanto à cifose nos portadores de fraturas do subtipo B21 de Magerl, de acordo com a variação das médias entre os indivíduos após acompanhamento mínimo de seis meses. Métodos: estudo retrospectivo com 34 pacientes submetidos à realização de gesso antigravitacional ou colete TLSO. A mensuração da cifose foi realizada pelo método de Cobb no

\begin{abstract}
Introduction: the post-traumatic kyphosis is a complication reported after conservative treatment of thoracolumbar flexion fracture. Most of the series evaluates all subtypes of Magerl and discusses their findings based on the average loss of kyphosis during the follow-up. Objective: to evaluate radiographic outcomes regarding kyphosis in patients with fractures of Magerl subtype B21 according to the average variation among individuals after follow-up of at least six months. Methods: a retrospective study with 34 patients treated with hyperextension cast or TLSO brace. The measurement of kyphosis was performed by means of Cobb method at the admission
\end{abstract}

\section{RESUMEN}

Introducción: la cifosis postraumática es una complicación del tratamiento conservador de la fractura toracolumbar tipo flexión-distracción. La mayoría de las series evalúan todos los subtipos de Magerl y discuten sus resultados con base en la pérdida promedio de cifosis en el acompañamiento. Objetivo: evaluación del desenlace radiográfico según la cifosis en los portadores de fracturas del subtipo B21 de Magerl, de acuerdo con la variación de los promedios entre los individuos después de un acompañamiento mínimo de seis meses. Métodos: estudio retrospectivo con 34 pacientes sometidos a la realización de yeso antigravitacional o chaleco TLSO. La medición de la cifosis fue realizada por el método de Cobb en

\footnotetext{
Trabalho realizado no Departamento de Ortopedia e Traumatologia da Faculdade de Ciências Médicas da Santa Casa de Misericórdia de São Paulo - FCMSCSP - São Paulo (SP), Brasil.

'Médico do Curso de Aperfeiçoamento do Grupo de Coluna do Departamento de Ortopedia e Traumatologia da Faculdade de Ciências Médicas da Santa Casa de São Paulo - FCMSCSP - São Paulo (SP), Brasil.

${ }^{2}$ Pós-graduando (Mestrado) do Departamento de Ortopedia e Traumatologia da Faculdade de Ciências Médicas da Santa Casa de São Paulo - FCMSCSP - São Paulo (SP), Brasil.

${ }^{3}$ Mestre; Professora Instrutora; Assistente do Grupo de Coluna do Departamento de Ortopedia e Traumatologia da Faculdade de Ciências Médicas da Santa Casa de São Paulo - FCMSCSP - São Paulo (SP), Brasil.

${ }^{4}$ Doutor; Professor Assistente e Chefe do Grupo de Coluna do Departamento de Ortopedia e Traumatologia da Faculdade de Ciências Médicas da Santa Casa de São Paulo - FCMSCSP - São Paulo (SP), Brasil.

${ }^{5}$ Doutor; Professor e Consultor do Grupo de Coluna do Departamento de Ortopedia e Traumatologia da Faculdade de Ciências Médicas da Santa Casa de São Paulo - FCMSCSP - São Paulo (SP), Brasil.

¿Doutor; Professor Adjunto e Diretor do Departamento de Ortopedia e Traumatologia da Faculdade de Ciências Médicas da Santa Casa de São Paulo - FCMSCSP São Paulo (SP), Brasil.
} 
momento da admissão no hospital e no final do acompanhamento médio de 84,5 meses. Resultados: não houve diferença estatística entre a cifose antes e após o tratamento $\left(14,18^{\circ}\right.$ versus $14,15^{\circ}$; $(\mathrm{p}=0,967)$. Conclusão: a cifose radiográfica no final do acompanhamento é similar à cifose encontrada nas radiografias iniciais nesse grupo de pacientes.

DESCRITORES: Fraturas da coluna vertebral/radiografia; Fraturas da coluna vertebral/ classificação; Cifose; Resultado de tratamento in the hospital and at the end of the follow-up of approximately 84.5 months. Results: there was no statistical difference between the kyphosis before and after treatment (14.18 versus $14.15^{\circ} ; \quad(p=0.967)$. Conclusion: the radiographic kyphosis in the final follow-up and in the initial radiographies is similar in this group of patients.

KEYWORDS: Spinal fractures/ radiography; Spinal fractures/ classification; Kyphosis; Treatment outcome el momento de La admisión en el hospital y en el final del acompañamiento promedio de 84,5 meses. Resultados: no hubo diferencia estadística entre la cifosis antes y después del tratamiento $\left(14,18^{\circ}\right.$ versus $14,15^{\circ} ; \quad(p=0,967)$. Conclusión: la cifosis radiográfica en el final del acompañamiento es similar a la encontrada en las radiografias iniciales en este grupo de pacientes.

DESCRIPTORES: Fracturas de la columna vertebral/radiografia; Fracturas de la columna vertebral/clasificación; Cifosis; Resultado del tratamiento

\section{INTRODUÇÃO}

As fraturas da região toracolombar são as mais frequentes do esqueleto axial e correspondem a $89 \%$ das fraturas da coluna vertebral ${ }^{1}$.

O número crescente de acidentes industriais e automobilísticos tem influência direta no aumento das lesões complexas e graves da coluna vertebral. Nos pacientes politraumatizados sua incidência é maior, bem como sua associação com lesões neurológicas. O tratamento dessas fraturas sofreu grandes modificações na última década, e o conhecimento das características individuais de cada tipo de fratura tem influenciado a estratégia terapêutica ${ }^{1-5}$.

Nos Estados Unidos, 11 mil novas lesões da medula espinal exigem tratamento a cada ano, sendo que 15 a $20 \%$ dos pacientes com fratura da coluna torácica e lombar sofrem lesão neurológica ${ }^{6}$. Em cada grupo de 1 milhão de habitantes, 40,1 sofrem trauma da coluna torácica ou lombar a cada ano. Isso resulta em uma despesa total ao governo, entre custos diretos e indiretos, de US $\$ 250.000,00$ por paciente por $\mathrm{ano}^{6}$.

$\mathrm{O}$ tratamento das fraturas da coluna toracolombar é tema controverso, especialmente quando o paciente não apresenta déficit neurológico, trazendo diferentes opiniões com relação às indicações do tratamento conservador ou cirúrgico ${ }^{7-9}$.

O tratamento cirúrgico tem sido indicado nos pacientes que apresentam lesão neurológica, estreitamento do canal vertebral superior a $50 \%$, redução da altura do corpo vertebral maior que $50 \%$ e cifose superior a $30^{\circ 7,10-15}$. Denis classificou as fraturas vertebrais baseando-se na teoria das três colunas e nos mecanismos que podem levar à falha das mesmas. Segundo seus critérios, o acometimento da coluna média acarreta instabilidade mecânica, muitas vezes passível de tratamento cirúrgico ${ }^{15}$.

O tratamento conservador está indicado para as fraturas que não apresentam as características de instabilidade do segmento vertebral mencionadas, que não mostram lesões neurológicas ou, ainda, em situações especiais em que existam contraindicações para o tratamento cirúrgico ${ }^{16}$.

Dentre as fraturas toracolombares, a lesão causada pelo mecanismo de flexão-distensão é infrequente e recebe denominações como fratura de Chance (FC), fratura do cinto de segurança $(F C S)^{17}$ e fratura flexão-distração (FFD). A fratura clássica descrita por Chance envolve uma divisão horizontal da vértebra, começando no processo espinhoso e lâminas, estendendo-se pelos pedículos e corpo vertebral, sem causar dano às estruturas ligamentares ${ }^{18}$. Desde então, muitas variantes desse tipo de fratura foram descritas ${ }^{19,20}$, e muita controvérsia tem ocorrido na tentativa tanto de denominar como de classificar tais fraturas ${ }^{21-23}$.

Em 1994, um abrangente sistema de classificação conhecido como AO (Arbeitsgemeinschaft für Osteosynthesefragen) foi introduzido por Magerl et al. ${ }^{24}$. O objetivo foi a avaliação dos aspectos morfológicos dos componentes dessas fraturas. Esses autores inferiram, inclusive, sobre as lesões das estruturas ligamentares a partir da análise de imagens de radiografias simples e de tomografia computadorizada. Os autores agruparam as lesões de acordo com sua morfologia e seus mecanismos de trauma. Essa classificação descreve três grupos principais de fraturas divididas por sua morfologia e diferentes mecanismos de trauma. Cada um desses grupos apresenta subgrupos em uma situação crescente de gravidade do trauma e de instabilidade mecânica. Assim, a classificação é composta por um total de 25 subtipos de fraturas, ordenados de acordo com a complexidade crescente das lesões, e que também incluem as fraturas do tipo flexo-distrativas nas suas diferentes manifestações.

Fraturas inicialmente classificadas como flexão-distração segundo os critérios de Denis puderam então ser interpretadas como fraturas associadas ao mecanismo de explosão quando aplicada a classificação de Magerl et al., isto é, apresentar 
além do comprometimento das estruturas posteriores a presença de fragmentos retropulsados para o canal vertebral.

Existe dúvida na literatura quanto ao comportamento dessas lesões, uma vez que existe associação de lesões ligamentares dos elementos posteriores com a fragmentação do corpo vertebral nas fraturas subtipo B21.

O objetivo deste estudo foi analisar retrospectivamente o colapso sagital na fratura toracolombar tipo B21 dos pacientes submetidos ao tratamento conservador.

\section{MÉTODOS}

Foi realizado estudo retrospectivo por meio da análise de prontuários do Serviço de Arquivos Médicos da Santa Casa de Misericórdia de São Paulo (SAME-SCMSP) do período compreendido entre 1997 e 2008 após aprovação do Comitê de Ética e Pesquisa em Seres Humanos do hospital. Um total de 61 pacientes com fratura toracolombar do tipo B foi tratado nesse período. Foram selecionados para estudo 34 pacientes portadores de fraturas B21, compreendidas entre T9 e L2, tratados de forma conservadora com confecção de gesso antigravitacional ou órtese TLSO (órtese toracolombosacral) em hiperextensão com seguimento mínimo de seis meses. Selecionamos pacientes sem lesão grave capsuloligamentar posterior. Para isso, utilizamos os critérios clínicos, como inexistência de dor à palpação no espaço correspondente aos processos espinhosos, e critérios radiográficos, como abertura interespinhosa do nível acometido como descrito por Newmann ${ }^{25}$. Foi definida como ponto de referência para a técnica de mensuração a porção superior da imagem em "lágrima" dos processos espinhosos identificada na radiografia na incidência em anteroposterior. A diferença maior ou igual a $20 \%$, em conjunto com os achados clínicos, foi considerada fratura tipo flexo-distrativa capsuloligamentar grave instável e, por isso, de indicação cirúrgica e excluída do estudo (Figura 1).

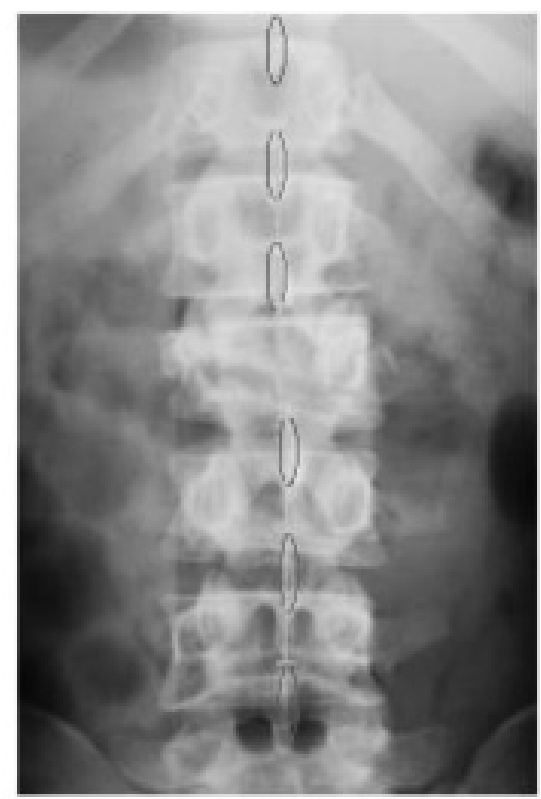

Figura 1

Ilustração do método de Neumann et al. ${ }^{25}$. A linha tracejada indica o local da lesão.
Filmes de radiografias com incidência anteroposterior e de perfil e tomografia computadorizada (TC), cortes axiais, reconstrução e sagitais foram revistos. O déficit neurológico foi avaliado com base na escala de Frankel et al. ${ }^{26}$. Iniciouse o tratamento após o diagnóstico com a manobra de redução mediante tração e hiperextensão da região toracolombar com fulcro de apoio na mesa de tração ortopédica nos casos submetidos à imobilização gessada. Excluímos portadores de fraturas com mais de dez dias do trauma, pacientes que foram submetidos a tratamento cirúrgico como tratamento inicial, fraturas patológicas, lesões por arma de fogo, fraturas em duas ou mais vértebras ou com documentação incompleta.

$\mathrm{O}$ estudo foi baseado em medidas obtidas a partir das radiografias no plano sagital, feitas no período do diagnóstico, pós-colocação imediata do gesso e último seguimento ambulatorial por meio de mensuração pelo método descrito por $\mathrm{Cobb}^{27}$ (Figura 2). Os parâmetros estudados foram: encunhamento do corpo vertebral fraturado no plano frontal e sagital, cifose do segmento vertebral envolvido na lesão e perda da correção (corpo) (Figuras 2 e 3$)^{28}$.

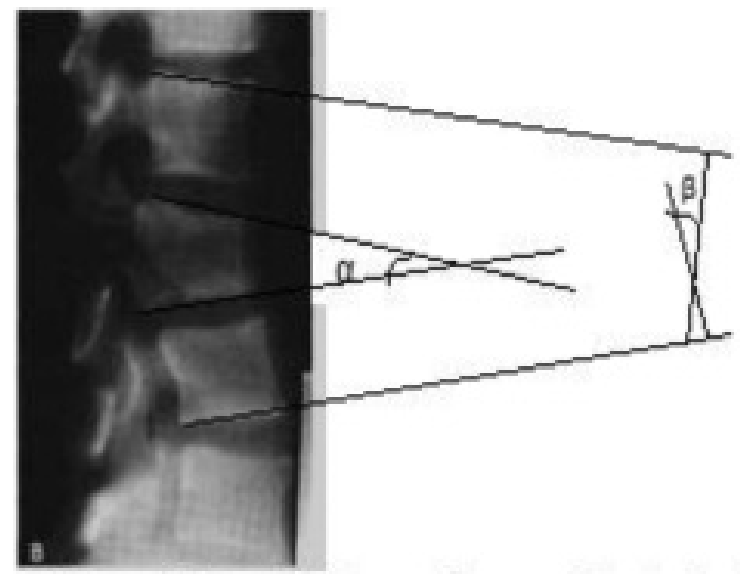

Figura 2

Desenho ilustrando a medida do ângulo de encunhamento do corpo vertebral (ângulo $\alpha$ ) e ângulo de cifose do segmento vertebral (ângulo $\beta$ ).
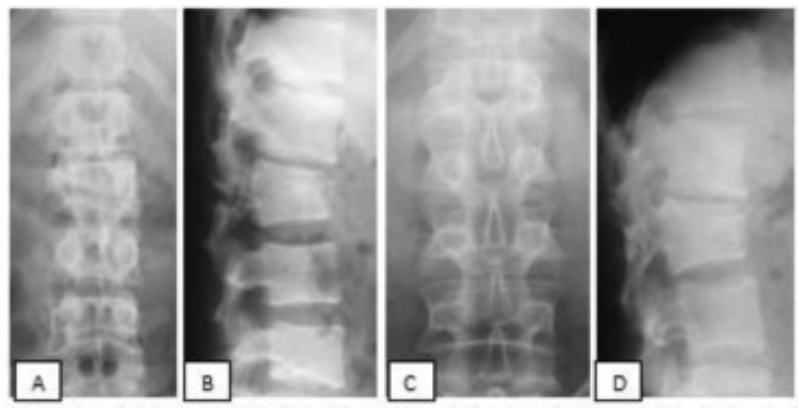

Figura 3

Paciente R.H.I., 39 anos, vítima de queda de altura com fratura tipo B21 em L2. Realizado tratamento conservador com gesso durante oito meses. A e B: Radiografias no ato do diagnóstico. C e D: Radiografias após 2 anos e 3 meses do tratamento inicial. 


\section{RESULTADOS}

Dos 34 pacientes, 9 (26,5\%) são do sexo feminino e 25 $(73,5 \%)$ são do sexo masculino (Gráfico 1$)$.

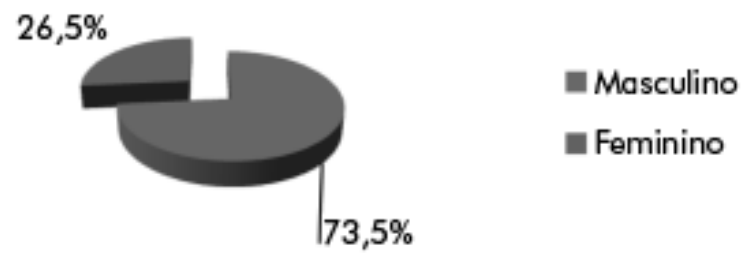

Gráfico 1

Distribuição dos pacientes em relação ao sexo.

A idade variou de 19 a 70 anos (média de 36,5 anos). Os mecanismos de trauma encontrados foram: queda de altura em 14 pacientes $(41,2 \%)$, acidente automobilístico em 9 (26,5\%), acidente motociclístico em 7 (20,6\%) e atropelamento em 4 (11,8\%) (Gráfico 2).

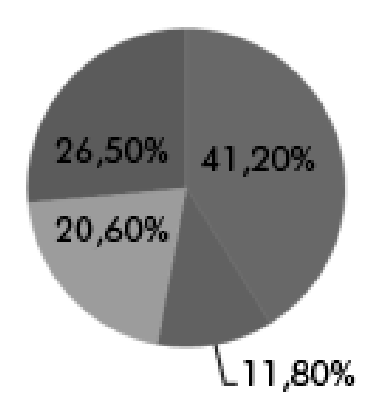

Queda de altura
Atropelamento
Moto
Automóvel

Gráfico 2

Relação percentual do mecanismo de trauma envolvido.

O tempo médio de internação foi de 9,61 dias com no mínimo de 3 e máximo de 35 dias. A vértebra T09 foi acometida em um paciente (2,9\%); T11, em um (2,9\%); T12, em três (8,8\%); L1, em $21(61,7 \%)$; e L2, em oito (23,5\%). O seguimento variou de 10 a 198 meses, com média de $84,59 \pm 48,615$ meses. Todos os pacientes foram classificados como E na Escala de Frankel durante todo o tratamento.

Foram 14 pacientes com lesões associadas, sendo um com fratura de antebraço, um com fratura de escafoide, um com fratura de punho, um com fratura de falange, um com fratura de tornozelo e luxação de quadril, um com pneumotórax, cinco com fratura de calcâneo, um com fratura de escápula, um com fratura de úmero e uma paciente com contusão pulmonar e sofrimento fetal, tendo de ser feita a retirada do feto com urgência.

A consolidação ocorreu em todos os pacientes e foi visualizada por meio de radiografias em anteroposterior e em perfil. Os valores mínimo e máximo da cifose da vértebra no inicio do tratamento foram de $4^{\circ}$ e $28^{\circ}$, respectivamente, com média de $14,18 \pm 5,926^{\circ}$. Os valores mínimo e máximo da cifose da vértebra no final do tratamento foram de $0^{\circ}$ e $36^{\circ}$, respectivamente, com média de $14,15 \pm 7,324^{\circ}$. Também foi medido o valor da cifose segmentar no período inicial do tratamento, que foi de $4^{\circ}$ e $32^{\circ}$, respectivamente, com média de $14,85 \pm 7,374^{\circ}$. O valor mínimo e o máximo da cifose segmentar no final do tratamento foram de $4^{\circ}$ e $33^{\circ}$, respectivamente, com média de $14,44 \pm 8,457^{\circ}$ (Tabela 1 , Gráficos 3 e 4 ). De todos os pacientes tratados, 38,5\% usaram somente aparelho gessado ou colete de Jewett, e $23,08 \%$ usaram os dois tipos de imobilização durante o tratamento. O tempo médio de imobilização foi de 7,08 meses, variando de 2 a 11 meses.

TABELA 1 - Resultados do tratamento

\begin{tabular}{|c|c|c|c|c|}
\hline Casos & $\begin{array}{l}\text { Cobb da } \\
\text { vértebra } \\
\text { inicial }\end{array}$ & $\begin{array}{c}\text { Cobb } \\
\text { segmentar } \\
\text { inicial }\end{array}$ & $\begin{array}{l}\text { Cobb da } \\
\text { vértebra } \\
\text { final }\end{array}$ & $\begin{array}{c}\text { Cobb } \\
\text { segmentar } \\
\text { final }\end{array}$ \\
\hline 1 & $14^{\circ}$ & $25^{\circ}$ & $19^{\circ}$ & $23^{\circ}$ \\
\hline 2 & $15^{\circ}$ & $10^{\circ}$ & $9^{\circ}$ & $7^{\circ}$ \\
\hline 3 & $15^{\circ}$ & $12^{\circ}$ & $11^{\circ}$ & $9^{\circ}$ \\
\hline 4 & $8^{\circ}$ & $16^{\circ}$ & $10^{\circ}$ & $18^{\circ}$ \\
\hline 5 & $11^{\circ}$ & $22^{\circ}$ & $12^{\circ}$ & $4^{\circ}$ \\
\hline 6 & $23^{\circ}$ & $26^{\circ}$ & $26^{\circ}$ & $27^{\circ}$ \\
\hline 7 & $24^{\circ}$ & $13^{\circ}$ & $20^{\circ}$ & $11^{\circ}$ \\
\hline 8 & $10^{\circ}$ & $21^{\circ}$ & $11^{\circ}$ & $6^{\circ}$ \\
\hline 9 & $10^{\circ}$ & $12^{\circ}$ & $8^{\circ}$ & $11^{\circ}$ \\
\hline 10 & $19^{\circ}$ & $14^{\circ}$ & $28^{\circ}$ & $24^{\circ}$ \\
\hline 11 & $8^{\circ}$ & $8^{\circ}$ & $10^{\circ}$ & $4^{\circ}$ \\
\hline 12 & $14^{\circ}$ & $16^{\circ}$ & $12^{\circ}$ & $14^{\circ}$ \\
\hline 13 & $28^{\circ}$ & $19^{\circ}$ & $20^{\circ}$ & $20^{\circ}$ \\
\hline 14 & $12^{\circ}$ & $20^{\circ}$ & $14^{\circ}$ & $22^{\circ}$ \\
\hline 15 & $24^{\circ}$ & $16^{\circ}$ & $36^{\circ}$ & $33^{\circ}$ \\
\hline 16 & $8^{\circ}$ & $5^{\circ}$ & $0^{\circ}$ & $4^{\circ}$ \\
\hline 17 & $18^{\circ}$ & $14^{\circ}$ & $16^{\circ}$ & $10^{\circ}$ \\
\hline 18 & $11^{\circ}$ & $10^{\circ}$ & $9^{\circ}$ & $10^{\circ}$ \\
\hline 19 & $14^{\circ}$ & $12^{\circ}$ & $15^{\circ}$ & $21^{\circ}$ \\
\hline 20 & $8^{\circ}$ & $4^{\circ}$ & $10^{\circ}$ & $6^{\circ}$ \\
\hline 21 & $12^{\circ}$ & $11^{\circ}$ & $12^{\circ}$ & $12^{\circ}$ \\
\hline 22 & $19^{\circ}$ & $30^{\circ}$ & $18^{\circ}$ & $27^{\circ}$ \\
\hline 23 & $12^{\circ}$ & $8^{\circ}$ & $8^{\circ}$ & $5^{\circ}$ \\
\hline 24 & $8^{\circ}$ & $6^{\circ}$ & $12^{\circ}$ & $9^{\circ}$ \\
\hline 25 & $26^{\circ}$ & $32^{\circ}$ & $27^{\circ}$ & $28^{\circ}$ \\
\hline 26 & $13^{\circ}$ & $7^{\circ}$ & $10^{\circ}$ & $5^{\circ}$ \\
\hline 27 & $17^{\circ}$ & $26^{\circ}$ & $16^{\circ}$ & $28^{\circ}$ \\
\hline 28 & $9^{\circ}$ & $7^{\circ}$ & $9^{\circ}$ & $8^{\circ}$ \\
\hline 29 & $10^{\circ}$ & $16^{\circ}$ & $6^{\circ}$ & $14^{\circ}$ \\
\hline 30 & $7^{\circ}$ & $10^{\circ}$ & $11^{\circ}$ & $14^{\circ}$ \\
\hline 31 & $16^{\circ}$ & $17^{\circ}$ & $18^{\circ}$ & $15^{\circ}$ \\
\hline 32 & $18^{\circ}$ & $22^{\circ}$ & $19^{\circ}$ & $24^{\circ}$ \\
\hline 33 & $17^{\circ}$ & $13^{\circ}$ & $15^{\circ}$ & $9^{\circ}$ \\
\hline 34 & $4^{\circ}$ & $5^{\circ}$ & $4^{\circ}$ & $9^{\circ}$ \\
\hline
\end{tabular}




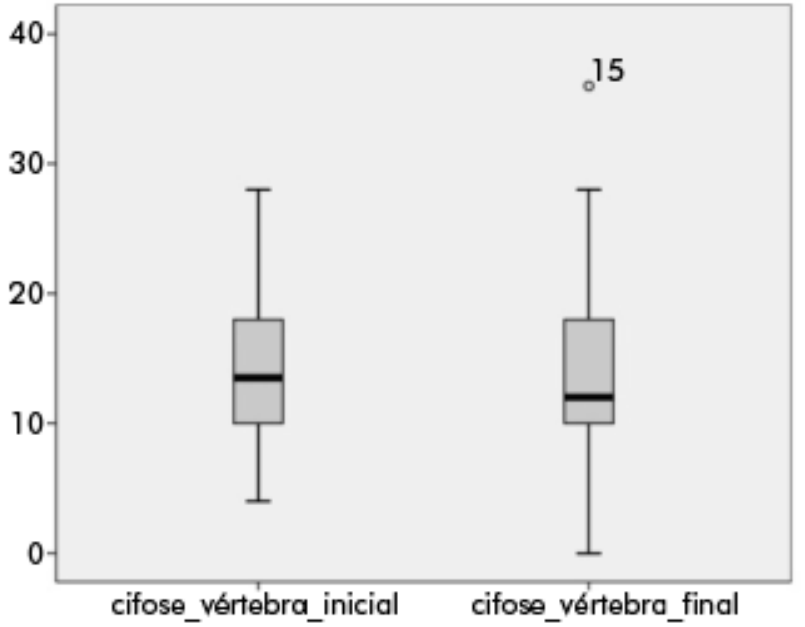

Gráfico 3

Cifose da vértebra.

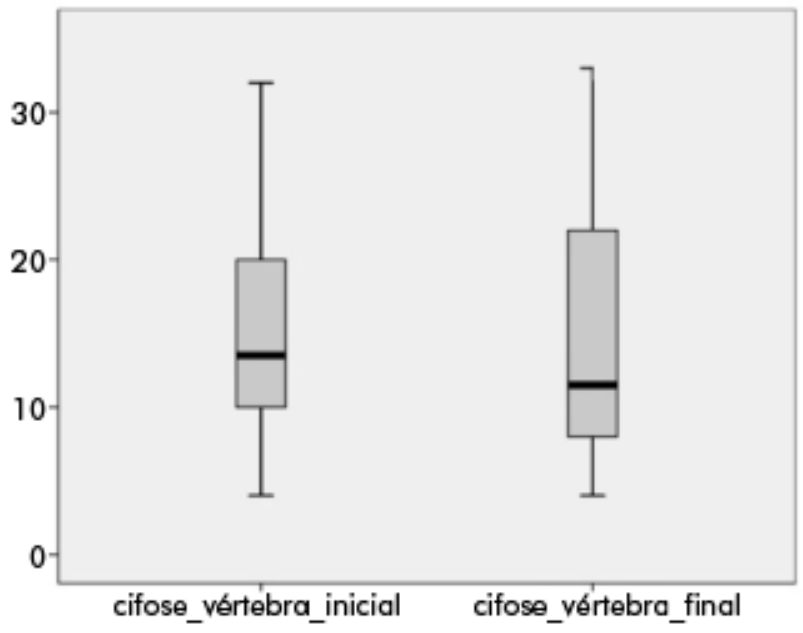

Gráfico 4

Cifose segmentar vertebral.

Foram encontradas duas complicações. Um paciente evoluiu com piora da cifose, sendo submetido a tratamento cirúrgico dois meses após o início do tratamento com colete de Jewett, e outro com dor intensa após nove meses de utilização de aparelho gessado em hiperextensão, também sendo feito tratamento cirúrgico após esse período.

Neste estudo, podemos observar que não houve significância estatística quando utilizamos o teste $t$ para comparar a cifose da vértebra inicial e ao final do acompanhamento $(p=0,967)$.

\section{DISCUSSÃO}

A fratura do tipo flexo-distrativa é uma das lesões as quais pode estar associada a falência das estruturas osteoligamentares da coluna vertebral por meio de um mecanismo de distração e flexão ${ }^{15,25,29,30}$. Todos os pacientes com suspeita de lesão por distração e flexão da coluna vertebral devem ser exaustivamente avaliados, uma vez que essas lesões são de difícil diagnóstico ${ }^{31-33}$.
Os mecanismos de trauma descritos são os de queda de altura e os acidentes de trânsito ${ }^{34-36}$. Por ser uma região com maior mobilidade do que a coluna torácica e lombar baixa, a transição toracolombar responde pela grande maioria dos casos em todas as séries dos trabalhos levantados, bem como em nossa casuística ${ }^{37-40}$. Houve um predomínio do sexo masculino nos achados do nosso trabalho, fato também encontrado nas demais publicações ${ }^{37,41,42}$.

As radiografias simples nas incidências anteroposterior e perfil são geralmente insuficientes para o diagnóstico, sendo características as alterações relativas à explosão da vértebra que compreendem a diminuição da altura do corpo vertebral e o alargamento do espaço entre os pedículos vertebrais. Porém, a lesão associada dos elementos posteriores pode ser suspeitada indiretamente pelo aumento da distância entre os processos espinhosos, visibilizado na incidência de frente ${ }^{32,33}$.

A análise prospectiva de pacientes submetidos ao tratamento conservador dessas lesões é rara na literatura. A maioria das séries aborda o resultado do tratamento cirúrgico. Em nosso meio, o tratamento conservador ainda é bastante empregado, principalmente nas lesões em que existe dúvida do real comprometimento das estruturas ligamentares. Associado a esse fato, a análise da fratura do corpo vertebral define lesões passíveis de tratamento conservador por não apresentar critérios mecânicos de instabilidade.

Com relação à dor, obtivemos apenas cinco pacientes com esse tipo de complicação ao final do tratamento, sendo que dois tiveram de ser submetidos ao tratamento cirúrgico para melhora do quadro. Possivelmente, esses pacientes eram portadores de lesão capsuloligamentar posteriores parciais não evidenciadas nas radiografias e TAC. Esses exames são de rotina no nosso serviço, entretanto, não sensíveis e específicos como a RM para averiguar comprometimento capsuloligamentar parcial posterior. A nosso ver, o acompanhamento periódico ambulatorial dessas pacientes pode prevenir a falta de tratamento nesse tipo de complicação. A RM, cabe destacar, não é estudo de diagnóstico de rotina nos pacientes portadores de fratura da coluna vertebral sem disfunção neurológica na maioria dos serviços hospitalares.

No nosso meio, Defino et al..$^{43}$ mostraram a experiência adquirida no tratamento conservador da fratura toracolombar em 64 casos. Um dos critérios de inclusão desse estudo foi a aderência ao tratamento com órtese; entretanto, esse dado da história coletada retrospectivamente pode não corresponder à realidade. De fato, esse aspecto é uma das limitações dos estudos retrospectivos.

Como podemos observar, as fraturas do tipo B sem lesão grave capsuloligamentar posterior e sem disfunção neurológica apresentam desfechos radiográficos satisfatórios após o tratamento conservador, de forma similar ao que já foi descrito por outros autores. Entretanto, alto índice de suspeita e acompanhamento periódico ambulatorial podem prevenir as complicações tardias do tratamento conservador das lesões 
instáveis pelo comprometimento capsuloligamentar posterior, muitas vezes não evidentes nas radiografias e $\mathrm{TAC}^{35,39}$.

\section{CONCLUSÃO}

No grupo de pacientes estudados, o tratamento conservador mostrou resultados satisfatórios após seguimento médio de
84,59 meses, devendo ser considerado como boa opção para o tratamento de fraturas toracolombares tipo B sem comprometimento neurológico ou lesão capsuloligamentar posterior.

O valor médio da cifose no início do tratamento se manteve relativamente constante no final do acompanhamento com o tratamento conservador.

\section{REFERÊNCIAS}

1. Herbert S, Xavier R, Junior Pardini AG, Barros Filho TE. Ortopedia e traumatologia, princípios e prática. $3^{\mathrm{a}}$ ed. São Paulo: Artmed; 2003.

2. Avanzi O, Salomão JCA, Dezen E, Pinto Filho WC. Fraturas da coluna vertebral em crianças: estudo de 38 casos. Rev Bras Ortop. 1993;28(3):105-11.

3. Puertas EB, Chagas JC, Mercúrio R, Milani A. Fraturas da coluna vertebral na região toracolombar: estudo de 36 pacientes. Rev Bras Ortop. 1991;26(4):196-200.

4. Solino JL, Melo MF, Silva DH, Elias N. Traumatismos da coluna vertebral: avaliação de etiologia, incidência e freqüência. Rev Bras Ortop. 1990;25(6):185-90.

5. Zardo E, Surmay MJ. Lesões da coluna vertebral. In: Hebert S, Xavier $\mathrm{R}$, editors. Ortopedia e traumatologia - Princípios e prática. Porto Alegre: Artes Médicas; 1995. cap. 26, p. 384401.

6. Bracken MB, Freeman DH Jr, Hellenbrand K. Incidence of acute traumatic hospitalized spinal cord injury in the United States, 1970-1977. Am J Epidemiol. 1981;113(6):615-22.

7. Cotrel Y, Dubousset J, Guillaumat M. New universal instrumentation for spinal surgery. Clin Orthop Relat Res. 1988;227:10-23.

8. Haher TR, Felmly WT, O'Brien N. Thoracic and lumbar fractures: diagnosis and management. In: Bridwell KH, DeWald RL, editors. The textbook of spinal surgery. Philadelphia: JB Lippincott; 1997.

9. Luque ER, Cassis N, Ramíres-Wiella G. Segmental spinal instrumentation in the treatment of fractures of the thoracolumbar spine. Spine (Phila $\mathrm{Pa}$ 1976). 1982;7(3):312-7.

10.Bohlman HH. Treatment of fractures and dislocations of the thoracic and lumbar spine. J Bone Joint Surg Am. 1985;67(1):165-9.
11. Bradford DS, Akbarnia BA, Winter RD, Seljeskog EC. Surgical stabilization of fractures and fracturedislocations of the thoracic spine. Spine (Phila Pa 1976). 1977;2(2):18596.

12.Levine AM, Edwards CC. Low lumbar burst fractures. Reduction and stabilization using the modular spine fixation system. Orthopedics. 1988;11(10):1427-32.

13.Akbarnia BA, Crandall DG, Burkus $\mathrm{K}$, Matthews T. Use of long rods and a short arthrodesis for burst fractures of the thoracolumbar spine. A long-term follow-up study. J Bone Joint Surg Am. 1994;76(11):1629-35.

14.Akalm S, Kis N, Benli IT, Citak M, Mumcu EF, Tüzüner M. Results of the AO spinal internal fixator in the surgical treatment of thoracolumbar burst fractures. Eur Spine J. 1994;3(2):102-6.

15.Denis F. The three column spine and its significance in the classification of acute thoracolumbar spinal injuries. Spine (Phila Pa 1976). 1983;8(8):81731.

16. Bedbrook GM. Treatment of thoracolumbar dislocation and fractures with paraplegia. Clin Orthop Relat Res. 1975;112:27-43.

17.Holt BW. Spines and seat belts: mechanisms of spinal injury in motor vehicle crashes. Med J Aust. 1976;2(11):411-3.

18. Chance GQ. Note on a type of flexion fracture of the spine. Br J Radiol. 1948;21(249):452.

19.Hall HE, Robertson WW Jr. Another Chance: a non-seatbelt related fracture of the lumbar spine. J Trauma. 1985;25(12):1163-6.

20.Howland WJ, Curry JL, Buffington CB. Fulcrum fractures of the lumbar spine. Transverse fracture induced by an improperly placed seat belt. JAMA. 1965;193:240-1.

21.Fuentes JM, Bloncourt J, Bourbotte G, Castan P, Vlahovitch B. La fracture de Chance. Neurochirurgie. 1984;30(2):113-8.
22.Korres DS, Nottis PG, Feroussis JC, Hartofilakidis-Garofalidis GK. La fracture de Chance et ses variants. Int Orthop. 1984;7(4):233-7.

23. Marsh HO, Bailey D. Seat belt fractures. Chance fractures caused by seat belts: presentation of three cases. J Kans Med Soc. 1970;71(9):361-5.

24.Magerl F, Aebi M, Gertzbein SD, Harms J, Nazarian S. A comprehensive classification of thoracic and lumbar injuries. Eur Spine J. 1994;3(4):184201.

25.Neumann P, Wang Y, Kärrholm J, Malchau H, Nordwall A. Determination of inter-spinous process distance in the lumbar spine. Evaluation of reference population to facilitate detection of severe trauma. Eur Spine J. 1999;8(4):272-8.

26.Frankel HL, Hancock DO, Hyslop G, Melzak J, Michaelis LS, Ungar GH, et al. The value of postural reduction in the initial management of closed injuries of the spine with paraplegia and tetraplegia. I. Paraplegia. 1969;7(3):179-92.

27.Cobb JR. Outline for the study of scoliosis. Instr Course Lect. 1948;5:261-75.

28.Defino HLA, Fuentes AER, Zatiti SCA, Engel EE. Avaliação da perda da correção no tratamento cirúrgico das fraturas da coluna torácica e lombar. Rev Bras Ortop. 1993;28(8):553-8 .

29.Rennie W, Mitchell N. Flexion distraction fractures of the thoracolumbar spine. J Bone Joint Surg Am. 1973;55(2):386-90.

30. Smith WS, Kaufer H. Patterns and mechanisms of lumbar injuries associated with lap seat belts. J Bone Joint Surg Am. 1969; 51(2): 239-54.

31. Coelho DG, Brasil AVB. Fratura do cinto de segurança da coluna lombar. Rev Bras Ortop. 1997; 32(2)100-1.

32.LeGay DA, Petrie DP, Alexander DI. Flexio-distraction injuries of the lumbar spine and associated abdominal trauma. J Trauma. 1990;30(4):436-44. 
33.Puno, MR, Bhoraj SY, Glassman SD. Flexion-distraction injuries of the thoracolumbar and lumbar spine in the adult and pediatric patient: seat belt injuries. Spine (Phila Pa 1976). 1993;7:223-40.

34. Saal JS. General principles of diagnostic testing as related to painful lumbar spine disorders: a critical appraisal of current diagnostic techniques. Spine (Phila Pa 1976). 2002;27(22):2538-45.

35.Anderson PA, Henley MB, Rivara, FP, Maier RV. Flexion distraction and chance injuries to the thoracolumbar spine. J Orthop Trauma. 1991;5(2):153-60.

36. Cantor JB, Lebwohl NH, Garvey T, Eismont FJ. Nonoperative management of stable thoracolumbar burst fractures with early ambulation and bracing. Spine (Phila Pa 1976). 1993;18(8):971-6.
37.Mumford J, Weinstein JN, Spratt KF, Goel VK. Thoracolumbar burst fractures. The clinical efficacy and outcome of nonoperative management. Spine (Phila Pa 1976). 1993;18(8):955-70.

38.Krompinger WJ, Fredrickson BE, Mino DE, Yuan HA. Conservative treatment of fractures of the thoracic and lumbar spine. Orthop Clin North Am. 1986;17(1):161-70.

39. Wood K, Buttermann G, Mehbod A, Garvey T, Jhanjee R, Sechriest V, et al. Operative compared with nonoperative treatment of a thoracolumbar burst fracture without neurological deficit. A prospective, randomized study. J Bone Joint Surg Am. 2003;85-A(5):773-81.

40.Shen WJ, Shen YS. Nonsurgical treatment of three-column thoracolumbar junction burst fractures without neurologic deficit. Spine (Phila Pa 1976). 1999;24(4):412-5.
41.Cunha FM, Menezes CM, Guimarães EP. Lesões traumáticas da coluna torácica e lombar. Rev Bras Ortop. 2000;35(1/2):17-22.

42.Jorge FF, Baldani APS, Barros Filho TP. Trauma raquimedular: aspectos epidemiológicos, impacto social e prevenção. Acta Ortop Bras. 1995;3(1):1-4.

43.Defino HLA, Fuentes ARR, Remondi PH, Ballim EC. Tratamento conservador das fraturas da coluna toracolombar. Rev Bras Ortop. 2000;35(8):301-8.

\section{Correspondência}

Robert Meves

Rua Dr. Cesário Motta Jr., 112

CEP 01221 -020 - São Paulo (SP), Brasil

E-mail: robertmeves@hotmail.com 